\title{
Student Perceptions of the Ophthalmology Curriculum in Medical School
}

\author{
Lucy Cobbs, BA ${ }^{1}$ Edmund Tsui, MD ${ }^{2}$ llyse D. Haberman, MD $^{2}$ Eleanore Kim, MD ${ }^{2}$
} Laurence Sperber, MD ${ }^{2}$ Mengfei $\mathrm{Wu}, \mathrm{MS}^{2}$ Joel S. Schuman, MD²,3,4

\footnotetext{
${ }^{1}$ New York University School of Medicine, New York, New York

2 Department of Ophthalmology, New York University School of Medicine, New York, New York

3 Department of Neuroscience and Physiology, NYU Langone Medical Center, NYU School of Medicine, New York, New York

${ }^{4}$ Department of Electrical and Computer Engineering, NYU Tandon School of Engineering, Brooklyn, New York
}

\begin{abstract}
Address for correspondence Joel S. Schuman, MD, Department of Ophthalmology, NYU Langone Eye Center, New York University School of Medicine, 222 East 41st Street, Suite 463, New York, NY 10017 (e-mail: joel.schuman@nyu.edu).
\end{abstract}

Journal of Academic Ophthalmology 2018;10:e79-e82.

\begin{abstract}
Keywords

- medical school

- education

- ophthalmology

- curriculum

Purpose There is no national standard for ophthalmology curricula in U.S. and Canadian medical schools. We aim to evaluate medical students' perceptions of the current ophthalmology curriculum without mandatory rotation at New York University School of Medicine (NYUSOM).

Design A cross-sectional Internet survey.

Methods A cross-sectional Internet survey was distributed to all NYUSOM students in March to May 2017. The main parameters measured in the study were students' selfreported confidence with ophthalmology skills and satisfaction with curriculum.

Results Response rate was $27.5 \%$ (166 of 604) of NYUSOM students. Many students reported they were not comfortable diagnosing eye emergencies (64\%), using a direct ophthalmoscope (71\%), or testing visual acuity (50\%). The majority of students did not want ophthalmology to become a mandatory rotation, but reported additional inperson training would be most helpful, compared with videos, web-based didactics, lectures, or virtual training. Completion of an ophthalmology elective and more hours of ophthalmology training were associated with increased confidence with eye examination and greater satisfaction with the curriculum.

Conclusions It is critical for all physicians-in-training to have adequate skills in eye examination. Identifying areas of improvement and determining the best teaching modality will be important in updating the ophthalmology curriculum for medical students. The majority of medical students are not at all or only slightly confident with eye examinations. Increasing the amount of in-person ophthalmology training in medical school improves confidence with eye examination.
\end{abstract}

There is no national standard for ophthalmology curricula in U.S. and Canadian medical schools, and over the past 15 years, fewer schools are requiring ophthalmology rotations. In $2000,68 \%$ of schools required a clinical rotation ${ }^{1}$ and by 2012 , only $18 \%$ of schools held the requirement. ${ }^{2}$ In conjunc-

received

October 11, 2017 accepted after revision April 9, 2018 tion with this shift, studies show that the current state of ophthalmology training in medical school is insufficient in preparing students for residency. For example, first year family medicine residents have reported a lack of confidence performing ophthalmic clinical skills, ${ }^{3}$ fourth year medical
Copyright $@ 2018$ by Thieme Medical Publishers, Inc., 333 Seventh Avenue, New York, NY 10001, USA. Tel: +1(212) 584-4662.
License terms

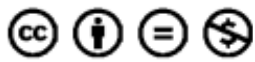


students fail to pass ophthalmology proficiency assessments, ${ }^{4}$ and eye examination skills taught only once during medical school erode without reinforcement. ${ }^{5}$

Ophthalmology exposure varies significantly between medical schools. The most recent comprehensive report on the state of ophthalmology education in the United States and Canada was a 2012-2013 survey study by the Association of University Professors of Ophthalmology (AUPO). Of the 109 schools surveyed, $84 \%$ reported training students in ophthalmic examination skills during preclinical education. ${ }^{2}$ Ophthalmology exposure during clinical training was more variable. ${ }^{2}$ All surveyed schools offered an elective ophthalmology rotation, and $72 \%$ of schools had a functional student interest group in ophthalmology, but a minority of the schools had a required clinical ophthalmology rotation. ${ }^{2}$

Despite the lack of emphasis on ophthalmology in many medical school curricula, eye examination and management skills are important for physicians to master because they can reveal systemic pathology and require emergent treatment. ${ }^{6-9}$ In addition, there is evidence that medical students are interested in learning more ophthalmology during medical school. ${ }^{10,11}$ In the context of rapidly evolving medical school curricula, and lack of national ophthalmology standards, it is important to assess ophthalmology training adequacy. $^{12}$ There is strong precedent for using surveybased research as a tool for evaluating the medical school curriculum. ${ }^{13-16}$ In recent years, studies have demonstrated deficiencies in medical education in several clinical subspecialties, including otolaryngology, dermatology, and musculoskeletal medicine. This suggests a need for widespread evaluation of the medical school curriculum. ${ }^{15,17,18}$

We sought to assess medical students' perceptions of ophthalmology training at New York University School of Medicine (NYUSOM), where there is no mandatory ophthalmology clinical rotation. Similar to many other schools, NYUSOM currently trains students in clinical ophthalmology skills during preclinical years, has a student interest group, and offers an optional clinical rotation but does not require one. The purpose of this study is to determine how comfortable students are with specific ophthalmology clinical skills and types of training they would find most helpful. The results may help target specific areas of the ophthalmology curriculum for improvement at NYUSOM and other schools with similar curricula.

\section{Methods}

Investigators created a 30-question cross-sectional survey (see - Supplementary Material) to characterize NYUSOM students' perceptions of ophthalmology exposure in preclinical and clinical years. The survey was designed in consultation with NYUSOM ophthalmologists, including the department chair, program director, medical student education director, and other faculty with extensive background in teaching. Questions aimed to explore students' satisfaction with the school's ophthalmology curriculum and extracurricular opportunities, confidence in their own ophthalmology clinical skills, and preferred teaching methods. Basic demographic information was also collected, including respondent's medical school class, gender, age, and preference for a surgical versus nonsurgical career. In March 2017, the survey was sent via email to all currently enrolled NYUSOM students, including those pursuing dual degrees. Email reminders were sent weekly, and the survey was closed in May 2017. Statistical analysis was performed using logistical regression for binary outcome measurements, and adjacent-category logit modeling for ordinal outcome measurements. Statistical significance was set at $p$-value $<0.05$. The study was approved by the Institutional Review Board and was HIPAA-compliant. Informed consent was obtained from participants, and the described research adhered to the tenets of the Declaration of Helsinki.

\section{Results}

Survey response rate was $27.5 \%$ (166 of 604) of NYUSOM students. Of the 166 respondents, there were 20 first years (12.0\%), 35 second years (21.1\%), 59 third years (35.5\%), 44 fourth years (26.5\%), and 8 students currently completing dual degrees (4.8\%). Thirty-seven percent of respondents were male and $63 \%$ were female. The majority of respondents preferred to enter a career with both medical and surgical aspects, while $11 \%$ preferred completely surgical and $28 \%$ preferred completely medical careers. Fifteen percent reported they were considering careers in ophthalmology.

The majority of students reported they were not satisfied with their ophthalmology training in medical school (83.7\%) and thought that the current curriculum does not provide adequate exposure to ophthalmology (91\%). Many reported they were not comfortable performing ophthalmology exam skills, including performing visual acuity testing (50\%), using a direct ophthalmoscope (71.1\%), performing a dilated eye exam (79.5\%), and identifying ocular emergencies (64.4\%). First-year medical students were more uncomfortable than greater class years, with $90 \%$ of MS1 students reporting being "very uncomfortable." However, the majorities of other classes also reported being uncomfortable, with 68.2\% MS4 students reporting being "very uncomfortable" or "uncomfortable"; $77.7 \%$ of respondents were "slightly confident" or "not at all confident" in their acquired ophthalmology skills. Gender was not significantly associated with lower confidence in eye examination skills. The majority of students reported that they thought ocular health is "important," “more important," or "very important” (97.7\%).

Several factors, including class year, taking the ophthalmology elective, and career aspirations, were associated with perception of training and confidence with clinical skills. Students who had not taken the optional ophthalmology elective were more likely to perceive their skill training as inadequate (odds ratio $(\mathrm{OR})=1.8, p=0.029)$. Students who had taken the elective were 3.85 times $(p=0.018)$ more likely to be satisfied with the curriculum's exposure to ophthalmology. Without adjusting for covariates, students with 1 more hour of ophthalmology training were 2.25 times more likely to be satisfied with the curriculum $(p=0.002)$. However, if we adjust for covariates, the association is no 


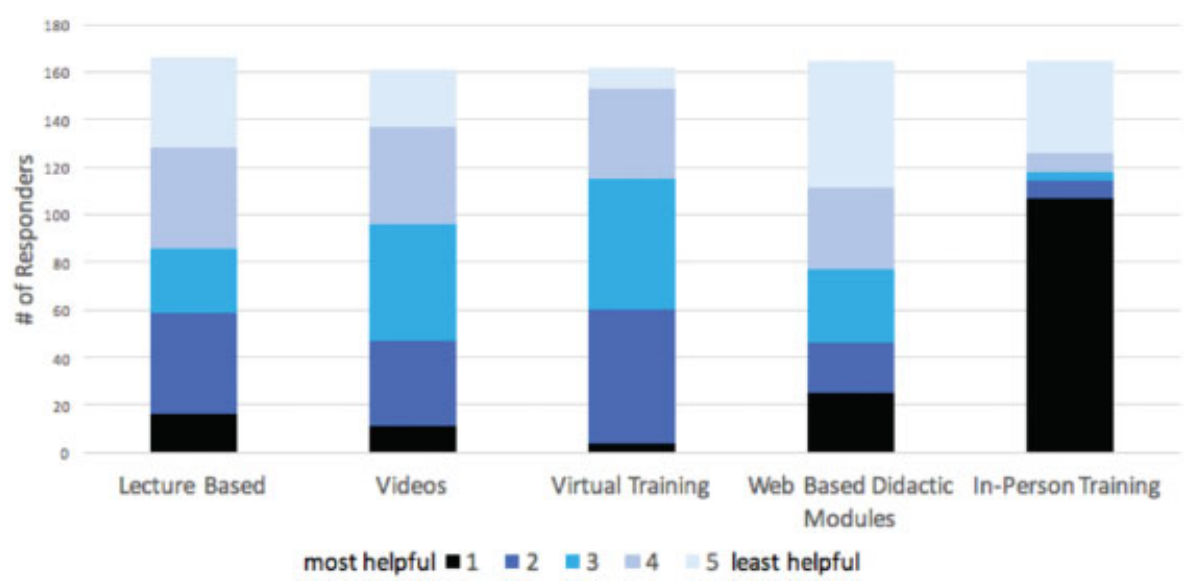

Fig. 1 Medical students ranked five ophthalmology training methods from most to least helpful. Most respondents wanted additional ophthalmology training to be in-person (107), followed by web-based didactic (25), lecture-based (16), video (11), and virtual ophthalmology training. Response rate was $97 \%$ (161/166) for ranking training methods.

longer significant. Five factors were associated with students' greater confidence in ophthalmology skills, including having taken the elective $(\mathrm{OR}=2.51, p=0.001)$, having more hours of ophthalmology training $(\mathrm{OR}=1.42, p=0.006)$, considering ophthalmology as a career $(\mathrm{OR}=2.03, p=0.012)$, having no family history of ocular disease $(\mathrm{OR}=1.93$, $p=0.004$ ), and having experience caring for eye disease ( $\mathrm{OR}=1.58, p=0.039$ ). Three factors were associated with greater comfort in identifying ocular emergencies, including having more medical education ( $\mathrm{OR}=2.12, p=0.032)$, considering a career in ophthalmology $(\mathrm{OR}=1.97, p=0.028)$, and having cared for eye disease $(\mathrm{OR}=1.57, p=0.028)$. Four factors were associated with perceiving ocular health as important, including having taken the elective $(\mathrm{OR}=2.27$, $p=0.035)$, having more medical education ( $\mathrm{OR}=1.28$, $p=0.047$ ), wanting to pursue a more surgical career (OR $=1.19, p=0.029)$, and having shadowed an ophthalmologist $(\mathrm{OR}=1.77, p=0.037)$.

In terms of preference for additional ophthalmology education, the majority of students did not think there should be a required ophthalmology rotation (80.7\%). Most students reported they would find additional in-person ophthalmology training to be most helpful (64.8\%), followed by web-based didactic (15.2\%), lecture-based (9.6\%), video (6.8\%), and virtual ophthalmology training (2.5\%; - Fig. 1).

\section{Discussion}

Our survey results are largely consistent with previous studies' findings on ophthalmology education; however, we also explore additional factors associated with confidence in ophthalmology clinical skills. NYUSOM students reported greater comfort with visual acuity testing than with dilated eye and direct ophthalmoscope examinations. Similarly, in a previous study, University of Massachusetts' medical school students self-reported more confidence with visual acuity testing than with dilated eye examination. ${ }^{19}$ Almost all (97.5\%) of U.S. medical schools surveyed in a previous study reported they teach visual acuity assessment, but less than $60 \%$ teach dilated eye exam skills. ${ }^{19}$
Greater confidence in exam skills was associated with more hours of training and having taken the optional ophthalmology elective. Previous studies have similarly shown electives and more training time are associated with both students' and residents' improved perceptions of their own skills and their objective performance on assessments of ophthalmology knowledge. ${ }^{3,10}$ While more training hours are associated with greater confidence in clinical skills, the relationship between hours of education and skill proficiency may in fact be more complicated. Previous studies have shown that different training methods vary in their efficacy, ${ }^{3,11,20}$ and our study showed that students prefer certain training methods, such as in-person education, over others, including video didactics. Although previous studies have shown varying success between training methods, there is no consensus as to what type of education is most effective. In addition to training method, timing of education is important, and training distributed across all 4 years of medical education may be more effective than training isolated to 1 or 2 years of medical education., ${ }^{4,5}$ A third factor important for education and assessing students' competence is feedback. NYUSOM provides live feedback to students during the preclinical teaching session to improve eye examination skills, and other forms of feedback may include written assessments, objective structured clinical examinations, and simulations. Therefore, the training method, timing, and feedback mechanism, in addition to total number of hours, should be carefully considered when revising ophthalmology curricula.

Our study was unique in that it identified additional factors besides hours of training and participation in an elective which were associated with clinical skills, confidence, and curriculum satisfaction. Factors related to personal interest in ophthalmology, including having shadowed an ophthalmologist and pursuing a career in ophthalmology, correlated with students valuing ocular health or having more confidence in their own clinical skills. In addition, having cared for someone with an eye disease was associated with greater confidence in ophthalmology skills. Therefore, introducing students to patients with ocular diseases and aiming to pique students' interest in 
ophthalmology, rather than formal skill training, may be an important component of an adequate education. Exposing students to ophthalmology early in the course of their medical education may motivate them to pursue more training throughout their education, leading to greater confidence and satisfaction with the curriculum.

Although NYUSOM students reported that additional inperson ophthalmology training would be most helpful, the majority of students did not want a required ophthalmology rotation. Therefore, changes to the curriculum should focus on integrating ophthalmology education into other rotations and preclinical learning, with an emphasis on early exposure, to demonstrate the importance of ocular health and inspire students to seek out shadowing and elective opportunities themselves. Given students' lack of comfort with direct ophthalmoscope and dilated eye exams in comparison with visual acuity exam, additional training should focus on these two skill sets. Also, to satisfy students' desire for inperson training and their low comfort with identifying ocular emergencies, a simulation may be beneficial and could be incorporated at many different points in the curriculum, including preclinical or clinical neurology, emergency medicine, or medicine rotations. Although our study did not parse diagnostic versus management knowledge, a previous cross-sectional study showed that students' diagnostic knowledge significantly surpassed management knowledge. ${ }^{4}$ With the aim of preparing students for residency, training should emphasize management skills and knowing when to refer patients to ophthalmologists.

Our study was limited by the fact that we did not compare NYUSOM students' perceptions to those of students at a school with a required ophthalmology rotation. Given this, we could not isolate the impact a required rotation may have on students' confidence and satisfaction. However, our results provide insight into ophthalmology exposure in medical school and indicate that curriculum adjustments may improve students' comfort with clinical skills. Future studies should explore the role of the required rotation, compare the efficacies of different training methods for ophthalmology, and determine whether there are crucial aspects of the curriculum that should be standardized. In addition, submitting this survey to other medical schools could make these results more generalizable.

\section{Funding}

None.

Conflict of Interest

None declared.

\section{References}

1 Quillen DA, Harper RA, Haik BG. Medical student education in ophthalmology: crisis and opportunity. Ophthalmology 2005; 112(11):1867-1868
2 Shah M, Knoch D, Waxman E. The state of ophthalmology medical student education in the United States and Canada, 2012 through 2013. Ophthalmology 2014;121(06):1160-1163

3 Chan TY, Rai AS, Lee E, Glicksman JT, Hutnik CM. Needs assessment of ophthalmology education for primary care physicians in training: comparison with the International Council of Ophthalmology recommendations. Clin Ophthalmol 2011; 5:311-319

4 Esparaz ES, Binder SB, Borges NJ. How prepared are medical students to diagnose and manage common ocular conditions. J Educ Eval Health Prof 2014;11:29

5 Lippa LM, Boker J, Duke A, Amin A. A novel 3-year longitudinal pilot study of medical students' acquisition and retention of screening eye examination skills. Ophthalmology 2006;113(01):133-139

6 Stern GA; The Association of University Professors of Ophthalmology Education Committee. Teaching ophthalmology to primary care physicians. Arch Ophthalmol 1995;113(06): 722-724

7 Yusuf IH, Salmon JF, Patel CK. Direct ophthalmoscopy should be taught to undergraduate medical students-yes. Eye (Lond) 2015; 29(08):987-989

8 Sussman EJ, Tsiaras WG, Soper KA. Diagnosis of diabetic eye disease. JAMA 1982;247(23):3231-3234

9 Levin AV, Christian CW; Committee on Child Abuse and Neglect, Section on Ophthalmology. The eye examination in the evaluation of child abuse. Pediatrics 2010;126(02):376-380

$10 \mathrm{Wu}$ DJ, Greenberg PB. A self-directed preclinical course in ophthalmic surgery. J Surg Educ 2016;73(03):370-374

11 Gupta RR, Lam W-C. Medical students' self-confidence in performing direct ophthalmoscopy in clinical training. Can J Ophthalmol 2006;41(02):169-174

12 Clarkson JG. Training in ophthalmology is critical for all physicians. Arch Ophthalmol 2003;121(09):1327

13 Lockwood JH, Sabharwal RK, Danoff D, Whitcomb ME. Quality improvement in medical students' education: the AAMC medical school graduation questionnaire. Med Educ 2004;38(03): 234-236

14 Pugnaire MP, Purwono U, Zanetti ML, Carlin MM. Tracking the longitudinal stability of medical students' perceptions using the AAMC graduation questionnaire and serial evaluation surveys. Acad Med 2004;79(10, Suppl):S32-S35

15 Ulman CA, Binder SB, Borges NJ. Assessment of medical students' proficiency in dermatology: Are medical students adequately prepared to diagnose and treat common dermatologic conditions in the United States? J Educ Eval Health Prof 2015;12:18

16 Slavin SJ, Schindler DL, Chibnall JT. Medical student mental health 3.0: improving student wellness through curricular changes. Acad Med 2014;89(04):573-577

17 Kuan EC, Badran KW, Passy V, Armstrong WB. Medical students comfort levels with performing the basic head and neck examination in practice: follow-up during the core clerkship year. J Surg Educ 2015;72(01):117-121

18 Day CS, Yeh AC, Franko O, Ramirez M, Krupat E. Musculoskeletal medicine: an assessment of the attitudes and knowledge of medical students at Harvard Medical School. Acad Med 2007; 82(05):452-457

19 Kleinberg TT, Kaushal S, Asdourian G. Ophthalmology Education in Medical School Curriculum Design: Assessing the Home Front. 2010. Available at: http://escholarship.umassmed.edu/ssp/106/. Accessed November 13, 2016

20 Gogate P, Deshpande M, Dharmadhikari S. Which is the best method to learn ophthalmology? Resident doctors' perspective of ophthalmology training. Indian J Ophthalmol 2008;56(05):409-412 